hep-ph/0604217

BA-06-13

\title{
Higgs Boson Mass from Orbifold GUTs with Split Supersymmetry
}

\author{
Ilia Gogoladze ${ }^{a 1}$, Tianjun Li ${ }^{b, c}$, V. N. Şenoğuz ${ }^{d}$ and Qaisar Shafi ${ }^{d}$ \\ ${ }^{a}$ Department of Physics and Astronomy, University of Delaware, Newark, DE 19716, USA \\ ${ }^{b}$ Department of Physics and Astronomy, Rutgers University, Piscataway, NJ 08854, USA \\ ${ }^{c}$ Institute of Theoretical Physics, Chinese Academy of Sciences, Beijing 100080, P. R. China \\ ${ }^{d}$ Bartol Research Institute, Department of Physics and Astronomy, University of Delaware, \\ Newark, DE 19716, USA
}

\begin{abstract}
We consider orbifold GUTs with $\mathcal{N}=1$ supersymmetry in which the Standard Model (SM) gauge couplings are unified at $M_{\mathrm{GUT}} \simeq 2 \times 10^{16} \mathrm{GeV}$ with one of the third family (charged) Yukawa couplings. With split supersymmetry the SM Higgs mass is estimated to be $131 \pm 10 \mathrm{GeV}$ for gauge-top quark Yukawa unification, which increases to $146 \pm 8 \mathrm{GeV}$ for gauge-bottom quark (or gauge-tau lepton) Yukawa unification.
\end{abstract}

\footnotetext{
${ }^{1}$ On a leave of absence from: Andronikashvili Institute of Physics, GAS, 380077 Tbilisi, Georgia.
} 


\section{Introduction}

In a recent paper [1] the Higgs boson mass of the Standard Model (SM) was estimated within the framework of 7-dimensional orbifold grand unified theories (7D orbifold GUTs) with $\mathcal{N}=1$ supersymmetry, compactified on the orbifold $M^{4} \times T^{2} / Z_{6} \times S^{1} / Z_{2}$ (for details see Appendix A and Refs. [1, 2, 3, 4]). It was assumed in 1] that the $4 \mathrm{D} \mathcal{N}=1$ supersymmetry (SUSY) is broken at $M_{\mathrm{GUT}}$ and, depending on the gaugeYukawa unification assumed, the Higgs mass varies between $135 \pm 6 \mathrm{GeV}$ and $144 \pm 4$ $\mathrm{GeV}$. The unification condition fixes $\tan \beta$ (ratio of the two Minimal Supersymmetric Standard Model (MSSM) VEVs) which makes the Higgs mass prediction possible.

In this paper we extend the discussion in [1] to the case of split supersymmetry in which the supersymmetric scalars are at an intermediate scale $m_{S}\left(\sim 10^{6}-10^{11} \mathrm{GeV}\right)$, while gauge coupling unification is achieved with TeV-scale gauginos and Higgsinos. As in the MSSM, the Higgs mass is determined by its quartic coupling $\lambda$ generated by the supersymmetric D-term at $m_{S}$ and the one-loop top quark Yukawa corrections to the Higgs potential at the weak scale. Unlike the MSSM where the Higgs mass prediction also depends on the soft supersymmetry breaking A terms and stop masses due to radiative corrections, in split supersymmetry the $\mathrm{A}$ terms are suppressed relative to

$m_{S}$, and the threshold corrections from the stops are tiny. As a result, the Higgs boson mass can be calculated quite reliably, although it depends on the unknown parameter $m_{S}$.

The plan of this paper is as follows. In Section 2 we summarize the $7 \mathrm{D} S U(7)$ orbifold models and show how gauge-Yukawa coupling unification can be realized. Section 3 is a brief review of split SUSY. Section 4 is devoted to the Higgs boson mass predictions. We conclude in Section 5.

\section{$2 \quad S U(7)$ Orbifold Models}

To realize the unification of gauge couplings and one of the Yukawa couplings for the third-family quarks and lepton at the GUT scale, we consider a 7D $\mathcal{N}=1$ supersymmetric $S U(7)$ gauge theory compactified on the orbifold $M^{4} \times T^{2} / Z_{6} \times S^{1} / Z_{2}$ (for some details see Appendix A). We find that $S U(7)$ is the smallest gauge group which allows us to implement these gauge-Yukawa coupling unification conditions.

The $\mathcal{N}=1$ supersymmetry in 7D has 16 supercharges corresponding to $\mathcal{N}=4$ supersymmetry in 4-dimension (4D), and only the gauge supermultiplet can be introduced in the bulk. This multiplet can be decomposed under $4 \mathrm{D} \mathcal{N}=1$ supersymmetry into a gauge vector multiplet $V$ and three chiral multiplets $\Sigma_{1}, \Sigma_{2}$, and $\Sigma_{3}$, all in the adjoint representation, where the fifth and sixth components of the gauge field, $A_{5}$ and $A_{6}$, are contained in the lowest component of $\Sigma_{1}$, and the seventh component of the 
gauge field $A_{7}$ is contained in the lowest component of $\Sigma_{2}$. As pointed out in Ref. [5] the bulk action in the Wess-Zumino gauge and in $4 \mathrm{D} \mathcal{N}=1$ supersymmetry notation contains trilinear terms involving the chiral multiplets $\Sigma_{i}$. Appropriate choice of the orbifold enables us to identify some of them as the SM Yukawa couplings 3 .

To break the $S U(7)$ gauge symmetry, we select the following $7 \times 7$ matrix representations for $R_{\Gamma_{T}}$ and $R_{\Gamma_{S}}$ defined in Appendix $\mathrm{A}$

$$
\begin{gathered}
R_{\Gamma_{T}}=\operatorname{diag}\left(+1,+1,+1, \omega^{n_{1}}, \omega^{n_{1}}, \omega^{n_{1}}, \omega^{n_{2}}\right), \\
R_{\Gamma_{S}}=\operatorname{diag}(+1,+1,+1,+1,+1,-1,-1),
\end{gathered}
$$

where $n_{1}$ and $n_{2}$ are positive integers, and $n_{1} \neq n_{2}$. Then, we obtain

$$
\begin{gathered}
\left\{S U(7) / R_{\Gamma_{T}}\right\}=S U(3)_{C} \times S U(3) \times U(1) \times U(1)^{\prime}, \\
\left\{S U(7) / R_{\Gamma_{S}}\right\}=S U(5) \times S U(2) \times U(1), \\
\left\{S U(7) /\left\{R_{\Gamma_{T}} \cup R_{\Gamma_{S}}\right\}\right\}=S U(3)_{C} \times S U(2)_{L} \times U(1)_{Y} \times U(1)_{\alpha} \times U(1)_{\beta} .
\end{gathered}
$$

So, the $7 \mathrm{D} \mathcal{N}=1$ supersymmetric gauge symmetry $S U(7)$ is broken down to $4 \mathrm{D} \mathcal{N}=1$ supersymmetric gauge symmetry $S U(3)_{C} \times S U(2)_{L} \times U(1)_{Y} \times U(1)_{\alpha} \times U(1)_{\beta}$ [4]. In Eq. (41) we see the appearance of two U(1) gauge symmetries which we assume can be spontaneously broken at or close to $M_{\mathrm{GUT}}$ by the usual Higgs mechanism. It is conceivable that these two symmetries can play some useful role as flavor symmetries, but we will not pursue this any further here. A judicious choice of $n_{1}$ and $n_{2}$ will enable us to obtain the desired zero modes from the chiral multiplets $\Sigma_{i}$ defined in Appendix A.

The $S U(7)$ adjoint representation 48 is decomposed under the $S U(3)_{C} \times S U(2)_{L} \times$ $U(1)_{Y} \times U(1)_{\alpha} \times U(1)_{\beta}$ gauge symmetry as:

$$
48=\left(\begin{array}{cccc}
(\mathbf{8}, \mathbf{1})_{Q 00} & (\mathbf{3}, \overline{\mathbf{2}})_{Q 12} & (\mathbf{3}, \mathbf{1})_{Q 13} & (\mathbf{3}, \mathbf{1})_{Q 14} \\
(\overline{\mathbf{3}}, \mathbf{2})_{Q 21} & (\mathbf{1}, \mathbf{3})_{Q 00} & (\mathbf{1}, \mathbf{2})_{Q 23} & (\mathbf{1}, \mathbf{2})_{Q 24} \\
(\overline{\mathbf{3}} \mathbf{1})_{Q 31} & (\mathbf{1}, \overline{\mathbf{2}})_{Q 32} & (\mathbf{1}, \mathbf{1})_{Q 00} & (\mathbf{1}, \mathbf{1})_{Q 34} \\
(\overline{\mathbf{3}} \mathbf{1})_{Q 41} & (\mathbf{1}, \overline{\mathbf{2}})_{Q 42} & (\mathbf{1}, \mathbf{1})_{Q 43} & (\mathbf{1}, \mathbf{1})_{Q 00}
\end{array}\right)+(\mathbf{1}, \mathbf{1})_{Q 00}
$$

where the $(\mathbf{1}, \mathbf{1})_{Q 00}$ in the third and fourth diagonal entries of the matrix and the last term $(\mathbf{1}, \mathbf{1})_{Q_{00}}$ denote the gauge fields associated with $U(1)_{Y} \times U(1)_{\alpha} \times U(1)_{\beta}$. The subscripts $Q i j$, which are anti-symmetric $(Q i j=-Q j i)$, are the charges under $U(1)_{Y} \times U(1)_{\alpha} \times U(1)_{\beta}$. The subscript $Q 00=(\mathbf{0}, \mathbf{0}, \mathbf{0})$, and the other subscripts $Q i j$ with $i \neq j$ will be given for each model explicitly. 


\subsection{Unification of Gauge and Top Quark Yukawa Coupling}

To achieve gauge and top quark Yukawa coupling unification at $M_{\mathrm{GUT}}$, we make the following choice

$$
n_{1}=5 \text { and } n_{2}=2 \text { or } 3,
$$

in Eq. (11). This allows us to obtain zero modes from $\Sigma_{i}$ corresponding to a pair of Higgs doublets $H_{u}$ and $H_{d}$, as well as the left- and right-handed top quark superfields.

\begin{tabular}{|c|c|}
\hline Chiral Fields & Zero Modes \\
\hline \hline$\Sigma_{1}$ & $Q_{3}:(\mathbf{3}, \overline{\mathbf{2}})_{Q 12}$ \\
\hline$\Sigma_{2}$ & $H_{u}:(\mathbf{1}, \mathbf{2})_{Q 23} ; H_{d}:(\mathbf{1}, \overline{\mathbf{2}})_{Q 32}$ \\
\hline$\Sigma_{3}$ & $t^{c}:(\overline{\mathbf{3}}, \mathbf{1})_{Q 31}$ \\
\hline
\end{tabular}

Table 1: Zero modes from the chiral multiplets $\Sigma_{1}, \Sigma_{2}$ and $\Sigma_{3}$ with gauge and top quark Yukawa coupling unification.

The generators for the gauge symmetry $U(1)_{Y} \times U(1)_{\alpha} \times U(1)_{\beta}$ are as follows:

$$
\begin{aligned}
T_{U(1)_{Y}} & \equiv \frac{1}{6} \operatorname{diag}(1,1,1,0,0,-3,0)+\frac{\sqrt{21}}{42} \operatorname{diag}(1,1,1,1,1,1,-6), \\
T_{U(1)_{\alpha}} & \equiv-\frac{\sqrt{21}}{2} \operatorname{diag}(1,1,1,0,0,-3,0)+\operatorname{diag}(1,1,1,1,1,1,-6), \\
T_{U(1)_{\beta}} & \equiv \operatorname{diag}(1,1,1,-2,-2,1,0),
\end{aligned}
$$

With a canonical normalization $\operatorname{tr}\left[T_{i}^{2}\right]=1 / 2$ of non-abelian generators, from Eq. (7) we find $\operatorname{tr}\left[T_{U(1)_{Y}}^{2}\right]=5 / 6$. We denote the $U(1)_{Y}, S U(2)_{L}$, and $S U(3)_{C}$ gauge couplings

as $g_{Y}, g_{2}$, and $g_{3}$, respectively. So, for $k_{Y} g_{Y}^{2}=g_{2}^{2}=g_{3}^{2}$ at the GUT scale, this gives us the canonical $U(1)_{Y}$ normalization $k_{Y}=5 / 3$.

The charge assignments $Q i j$ from Eq. (5i) are as follows:

$$
\begin{aligned}
& Q 12=\left(\frac{1}{6},-\frac{\sqrt{21}}{2}, 3\right), \quad Q 14=\left(\frac{1+\sqrt{21}}{6}, \frac{14-\sqrt{21}}{2}, 1\right), \\
& Q 13=\left(\frac{\mathbf{2}}{\mathbf{3}}, \mathbf{- 2} \sqrt{\mathbf{2 1}}, \mathbf{0}\right), \quad Q 23=\left(\frac{\mathbf{1}}{\mathbf{2}},-\frac{\mathbf{3} \sqrt{\mathbf{2 1}}}{\mathbf{2}},-\mathbf{3}\right), \\
& Q 24=\left(\frac{\sqrt{21}}{6}, 7,-2\right), \quad Q 34=\left(\frac{-3+\sqrt{21}}{6}, \frac{14+3 \sqrt{21}}{2}, 1\right) .
\end{aligned}
$$

Substituting Eq. (6) in Eqs. (11)-(2) and employing the $Z_{6} \times Z_{2}$ transformation properties Eqs. (36) -(39) for the decomposed components of the chiral multiplets $\Sigma_{i}$, 
we obtain the zero modes given in Table 1. We can identify them as a pair of Higgs doublets as well as the left- and right-handed top quark superfields, as desired.

From the trilinear term in the 7D bulk action the top quark Yukawa coupling is contained in the term

$$
\int d^{7} x\left[\int d^{2} \theta g_{7} Q_{3} t^{c} H_{u}+\text { h.c. }\right],
$$

where $g_{7}$ is the $S U(7)$ gauge coupling at the compactification scale, which for simplicity, we identify as $M_{\mathrm{GUT}}$. We will ignore brane localized gauge kinetic terms, which may be suppressed by taking $V M_{*} \gtrsim O(100)$, where $V$ denotes the volume of the extra dimensions and $M_{*}$ is the cutoff scale [6]. With these caveats we obtain the $4 \mathrm{D}$ gaugetop quark Yukawa coupling unification at $M_{\mathrm{GUT}}$

$$
g_{1}=g_{2}=g_{3}=y_{t}=g_{7} / \sqrt{V},
$$

where $g_{1} \equiv \sqrt{5 / 3} g_{Y}$, and $y_{t}$ is the top quark Yukawa coupling.

As far as the remaining SM fermions are concerned, we note that on the 3-brane at the $Z_{6} \times Z_{2}$ fixed point $(z, y)=(0,0)$, the preserved gauge symmetry is $S U(3)_{C} \times$ $S U(2)_{L} \times U(1)_{Y} \times U(1)_{\alpha} \times U(1)_{\beta}$. Thus, on the observable 3 -brane at $(z, y)=(0,0)$, we can introduce the first two families of the SM quarks and leptons, the right-handed bottom quark, the $\tau$ lepton doublet, and the right-handed $\tau$ lepton. The $U(1)_{\alpha} \times U(1)_{\beta}$ anomalies can be canceled by assigning suitable charges to the SM quarks and leptons. For example, under $U(1)_{\alpha} \times U(1)_{\beta}$ the charges for the first-family quark doublet and the right-handed up quark can be respectively $(\sqrt{\mathbf{2 1}} / \mathbf{2},-\mathbf{3})$ and $(-\mathbf{2} \sqrt{\mathbf{2 1}}, \mathbf{0})$, while the charges of remaining SM fermions are zero.

\subsection{Unification of Gauge and Down-Type Yukawa Couplings}

To realize gauge-bottom quark Yukawa coupling unification, we make the following choice in Eq. (11):

$$
n_{1}=5, n_{2}=2 \text { or } 3 \text {. }
$$

The identification of $U(1)_{Y}$ differs from the previous subsection. The generators of $U(1)_{Y} \times U(1)_{\alpha} \times U(1)_{\beta}$ are defined as follows:

$$
\begin{aligned}
T_{U(1)_{Y}} & \equiv-\frac{1}{6} \operatorname{diag}(0,0,0,1,1,-2,0)+\frac{\sqrt{7}}{21} \operatorname{diag}(1,1,1,1,1,1,-6) \\
T_{U(1)_{\alpha}} & \equiv 2 \sqrt{7} \operatorname{diag}(0,0,0,1,1,-2,0)+\operatorname{diag}(1,1,1,1,1,1,-6) \\
T_{U(1)_{\beta}} & \equiv \operatorname{diag}(1,1,1,-1,-1,-1,0) .
\end{aligned}
$$

Note that $k_{Y}$ is also $5 / 3$ in this case. 


\begin{tabular}{|c|c|}
\hline Chiral Fields & Zero Modes \\
\hline \hline$\Sigma_{1}$ & $Q_{3}:(\mathbf{3}, \overline{\mathbf{2}})_{Q 12}$ \\
\hline$\Sigma_{2}$ & $H_{d}:(\mathbf{1}, \mathbf{2})_{Q 23} ; H_{u}:(\mathbf{1}, \overline{\mathbf{2}})_{Q 32}$ \\
\hline$\Sigma_{3}$ & $b^{c}:(\overline{\mathbf{3}}, \mathbf{1})_{Q 31}$ \\
\hline
\end{tabular}

Table 2: Zero modes from the chiral multiplets $\Sigma_{1}, \Sigma_{2}$ and $\Sigma_{3}$ with gauge and bottom quark Yukawa coupling unification.

The corresponding charges $Q i j$ are:

$$
\begin{aligned}
& Q 12=\left(\frac{\mathbf{1}}{\mathbf{6}}, \mathbf{- 2} \sqrt{\mathbf{7}}, \mathbf{2}\right), \quad Q 13=\left(-\frac{\mathbf{1}}{\mathbf{3}}, \mathbf{4} \sqrt{\mathbf{7}}, \mathbf{2}\right), \\
& Q 14=\left(\frac{\sqrt{7}}{3}, 7,1\right), \quad Q 34=\left(\frac{1+\sqrt{7}}{3}, 7-4 \sqrt{7},-1\right), \\
& Q 24=\left(\frac{-1+2 \sqrt{7}}{6}, 7+2 \sqrt{7},-1\right), \quad Q 23=\left(-\frac{1}{2}, 6 \sqrt{7}, 0\right) .
\end{aligned}
$$

In Table 2, we present the zero modes from the chiral multiplets $\Sigma_{1}, \Sigma_{2}$ and $\Sigma_{3}$. We identify them as the left-handed doublet $\left(Q_{3}\right)$, one pair of Higgs doublets $H_{u}$ and $H_{d}$, and the right-handed bottom quark $b^{c}$. From the trilinear term in the 7D bulk action in Eq. (27) we obtain the bottom quark Yukawa coupling

$$
\int d^{7} x\left[\int d^{2} \theta g_{7} Q_{3} b^{c} H_{d}+\text { h.c. }\right] .
$$

Thus, at $M_{\mathrm{GUT}}$ we have

$$
g_{1}=g_{2}=g_{3}=y_{b}=g_{7} / \sqrt{V},
$$

where $y_{b}$ is the bottom quark Yukawa coupling to $H_{d}$.

Finally, to realize the gauge-tau lepton Yukawa coupling unification, we set

$$
n_{1}=4, n_{2}=3 ; \text { or } n_{1}=3, n_{2}=2 .
$$

The generators for $U(1)_{Y} \times U(1)_{\alpha} \times U(1)_{\beta}$ are as follows:

$$
\begin{aligned}
T_{U(1)_{Y}} & \equiv \frac{1}{2} \operatorname{diag}(0,0,0,0,0,1,-1)-\frac{\sqrt{7}}{42} \operatorname{diag}(4,4,4,-3,-3,-3,-3), \\
T_{U(1)_{\beta}} & \equiv-\frac{2 \sqrt{7}}{3} \operatorname{diag}(0,0,0,0,0,1,-1)-\frac{1}{3} \operatorname{diag}(4,4,4,-3,-3,-3,-3), \\
T_{U(1)_{\alpha}} & \equiv \operatorname{diag}(0,0,0,1,1,-1,-1) .
\end{aligned}
$$


The $U(1)_{Y} \times U(1)_{\alpha} \times U(1)_{\beta}$ charges $Q i j$ are

$$
\begin{array}{ll}
Q 12=\left(-\frac{\sqrt{7}}{6},-\frac{7}{3},-1\right), & Q 13=\left(-\frac{3+\sqrt{7}}{6},-\frac{7-2 \sqrt{7}}{3}, 1\right), \\
Q 23=\left(-\frac{1}{2}, \frac{2 \sqrt{7}}{3}, 2\right), & Q 14=\left(\frac{3-\sqrt{7}}{6},-\frac{7+2 \sqrt{7}}{3}, 1\right), \\
Q 24=\left(\frac{1}{2},-\frac{2 \sqrt{7}}{3}, 2\right), & Q 34=\left(1,-\frac{4 \sqrt{7}}{3}, 0\right) .
\end{array}
$$

The zero modes include the third-family left-handed lepton doublet $L_{3}$, one pair of Higgs doublets $H_{u}$ and $H_{d}$, and the right-handed tau lepton $\tau^{c}$. From the trilinear term in the $7 \mathrm{D}$ bulk action, we obtain the $\tau$ lepton Yukawa term

$$
\int d^{7} x\left[\int d^{2} \theta g_{7} L_{3} \tau^{c} H_{d}+\text { h.c. }\right] .
$$

Thus, at the $M_{\mathrm{GUT}}$, we have

$$
g_{1}=g_{2}=g_{3}=y_{\tau}
$$

where $y_{\tau}$ is the tau lepton Yukawa coupling.

\section{Split Supersymmetry}

The split SUSY proposal [7, 8] abandons light SUSY scalars as a solution to the gauge hierarchy problem. The MSSM scalars are all assumed to be at an intermediate scale, except one Higgs doublet which is fine-tuned to be light. The fermionic superpartners remain light, preserving the gauge coupling unification and dark matter candidate.

For convenience, we assume the squarks, sleptons, charged and pseudoscalar Higgs to be all degenerate at the scalar mass scale $m_{S}$. The particle content in the effective theory beneath $m_{S}$ consists of the SM Higgs doublet $\mathrm{H}$, as well as the Higgsinos and gauginos. The Lagrangian is given by

$$
\begin{aligned}
\mathcal{L}= & m^{2} H^{\dagger} H-\frac{\lambda}{2}\left(H^{\dagger} H\right)^{2}-\left[h_{u} \bar{q} u \epsilon H^{*}+h_{d} \bar{q} d H+h_{e} \bar{\ell} e H\right. \\
& +\frac{M_{3}}{2} \tilde{g}^{A} \tilde{g}^{A}+\frac{M_{2}}{2} \tilde{W}^{a} \tilde{W}^{a}+\frac{M_{1}}{2} \tilde{B} \tilde{B}+\mu \tilde{H}_{u}^{T} \epsilon \tilde{H}_{d} \\
& \left.+\frac{H^{\dagger}}{\sqrt{2}}\left(\tilde{g}_{u} \sigma^{a} \tilde{W}^{a}+\tilde{g}_{u}^{\prime} \tilde{B}\right) \tilde{H}_{u}+\frac{H^{T} \epsilon}{\sqrt{2}}\left(-\tilde{g}_{d} \sigma^{a} \tilde{W}^{a}+\tilde{g}_{d}^{\prime} \tilde{B}\right) \tilde{H}_{d}+\text { h.c. }\right],
\end{aligned}
$$

where $\epsilon=i \sigma_{2}$. 
The SM Higgs doublet arises from a linear combination of the Higgs doublets $H_{u}$ and $H_{d}$ in the MSSM: $H=-\cos \beta \epsilon H_{d}^{*}+\sin \beta H_{u}$. By matching the Lagrangian in Eq. (21) with the interaction terms of the Higgs doublets $H_{u}$ and $H_{d}$ in the MSSM, the coupling constants of the effective theory at the scale $m_{S}$ are obtained at tree level as follows:

$$
\begin{aligned}
\lambda\left(m_{S}\right)= & \frac{\frac{3}{5} g_{1}^{2}\left(m_{S}\right)+g_{2}^{2}\left(m_{S}\right)}{4} \cos ^{2} 2 \beta, \\
h_{u}\left(m_{S}\right)=y_{u}^{*}\left(m_{S}\right) \sin \beta, & h_{d, e}\left(m_{S}\right)=y_{d, e}^{*}\left(m_{S}\right) \cos \beta, \\
\tilde{g}_{u}\left(m_{S}\right)=g_{2}\left(m_{S}\right) \sin \beta, & \tilde{g}_{d}\left(m_{S}\right)=g_{2}\left(m_{S}\right) \cos \beta, \\
\tilde{g}_{u}^{\prime}\left(m_{S}\right)=\sqrt{\frac{3}{5}} g_{1}\left(m_{S}\right) \sin \beta, & \tilde{g}_{d}^{\prime}\left(m_{S}\right)=\sqrt{\frac{3}{5}} g_{1}\left(m_{S}\right) \cos \beta .
\end{aligned}
$$

One expects to have threshold corrections to these relations from integrating out the heavy scalars at the scale $m_{S}$. But the mechanism which splits the scalar and fermionic superpartners of the SM particles will inevitably suppress the $A$-terms, so, $A \ll m_{S}$ and there are no significant finite corrections from integrating out the supersymmetric scalar particles [7].

\section{Higgs Mass Predictions}

In this section, we will calculate the Higgs boson mass as a function of the scalar mass scale $m_{S}$, assuming the gauge-top quark, gauge-bottom quark, and gauge-tau lepton Yukawa coupling unification. Once $\tan \beta$ is fixed from gauge-Yukawa coupling unification, the Higgs mass can be determined by running down all the couplings from the above boundary conditions ${ }^{2}$.

In our numerical calculations, we use two-loop renormalization group equation (RGE) running for the gauge couplings and one-loop RGE running for the Yukawa and Higgs quartic couplings. And the relevant RGEs are given in the Appendix of Ref. [8]. Also, we use the $\overline{\mathrm{MS}}$ parameters: the fine structure constant $\alpha_{E M}^{-1}\left(M_{Z}\right)=127.918$ and the weak mixing angle $\sin ^{2} \theta_{W}\left(M_{Z}\right)=0.23120$ [10], the bottom quark mass $m_{b}\left(M_{Z}\right)=2.9 \mathrm{GeV}$, and the top quark pole mass $M_{\text {top }}$ (pole) $=172.5 \pm 2.3 \mathrm{GeV}$ [1], where $M_{Z}$ is the $Z$ boson mass. We first run the parameters from $M_{Z}$ up to $m_{S}$ and adjust them until the boundary conditions at $m_{S}$ in Eq. (22) are satisfied. The second step is running the gauge and Yukawa couplings up to $M_{\mathrm{GUT}}$ with SUSY RGEs, and adjust $\tan \beta$ until the relevant Yukawa coupling $\left(y_{t, b, \tau}\right)$ equals to the gauge couplings at $M_{\mathrm{GUT}}$.

For gauge-top quark Yukawa coupling unification with $m_{S}=10^{9} \mathrm{GeV}$, the SM gauge couplings (more precisely $\alpha_{i}^{-1}$ ) are plotted in Fig. [1, which also displays the

\footnotetext{
${ }^{2}$ Higgs boson mass in split SUSY was calculated in Refs. [7, 8, 9] for sample $\tan \beta$ values.
} 


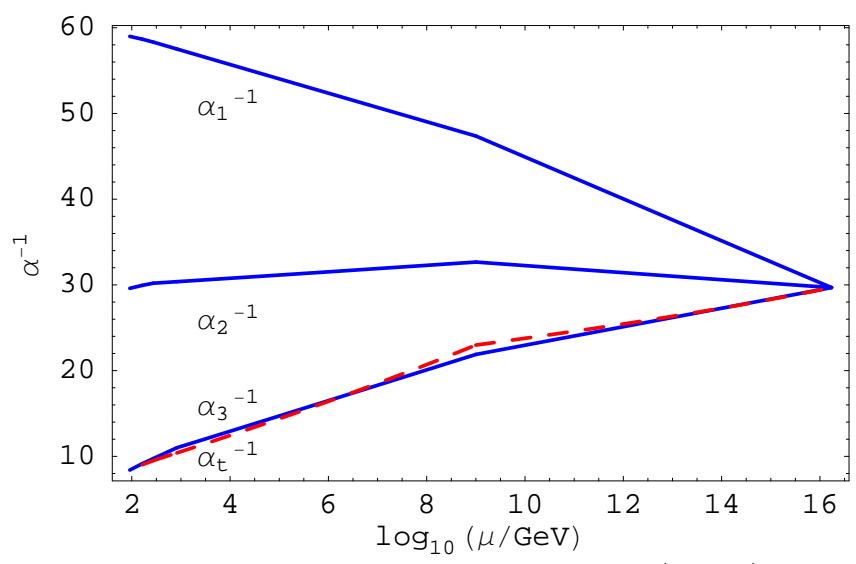

Figure 1: Two-loop RGE evolution of gauge couplings (solid) and one-loop RGE evolution of top quark Yukawa coupling (dashed), with $m_{S}=10^{9} \mathrm{GeV}$.

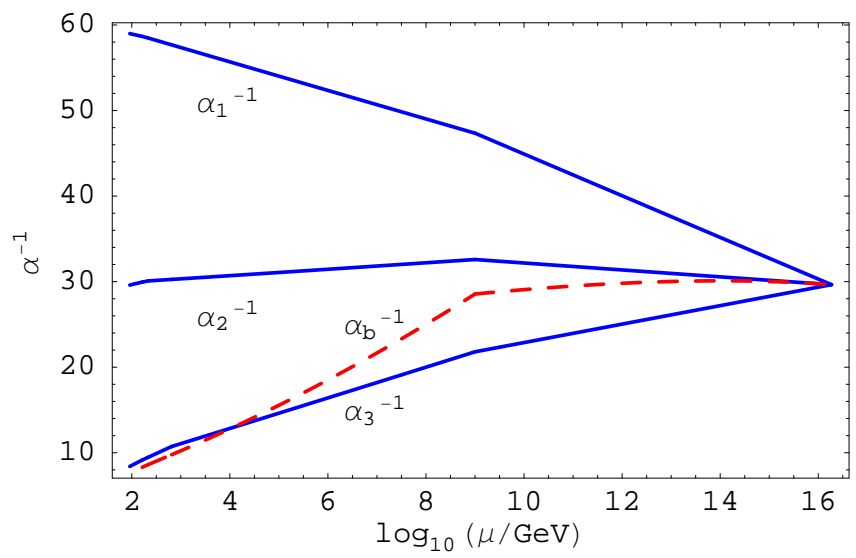

Figure 2: Two-loop RGE evolution of gauge couplings (solid) and one-loop RGE evolution of bottom quark Yukawa coupling (dashed), with $m_{S}=10^{9} \mathrm{GeV}$.

top quark Yukawa coupling $\alpha_{t}^{-1} \equiv 4 \pi / y_{t}^{2}$. And Fig. 2] shows the couplings for gaugebottom quark Yukawa coupling unification. In addition, $\tan \beta$ as a function of $m_{S}$ for gauge-top and gauge-bottom quark Yukawa coupling unification is given in Figs. 3 and 4. respectively. And $\tan \beta$ changes mildly with $m_{S}$ and remains $\sim 55$ for gauge-tau lepton Yukawa coupling unification.

Usually, $\alpha_{3}\left(M_{Z}\right)$ is taken as a free parameter to be determined by gauge coupling unification. In split SUSY, $\alpha_{3}\left(M_{Z}\right)$ decreases with increasing $m_{S}$, as expected. However, the value of $\alpha_{3}\left(M_{Z}\right)$ depends on the gaugino masses as well as $m_{S}$, decreasing as the gaugino masses increase [8, 12]. Let us denote $M_{1}, M_{2}, M_{3}$ as the bino, wino and gluino masses, respectively. For a simplified analysis, we assume $M_{1}=M_{2}=\mu=M_{1 / 2}$ where these masses refer to the running masses at $M_{1 / 2}$, and compare two cases with $M_{3} / M_{1 / 2}=3$ and $M_{3} / M_{1 / 2}=10$. The first case is typical for gaugino mass unification, while the second for anomaly mediated SUSY breaking [8]. $\alpha_{3}\left(M_{Z}\right)$ is considerably higher for the latter case with fixed $M_{1 / 2}$ [13. By fixing $\alpha_{3}\left(M_{Z}\right)=0.119 \pm 0.003$, 


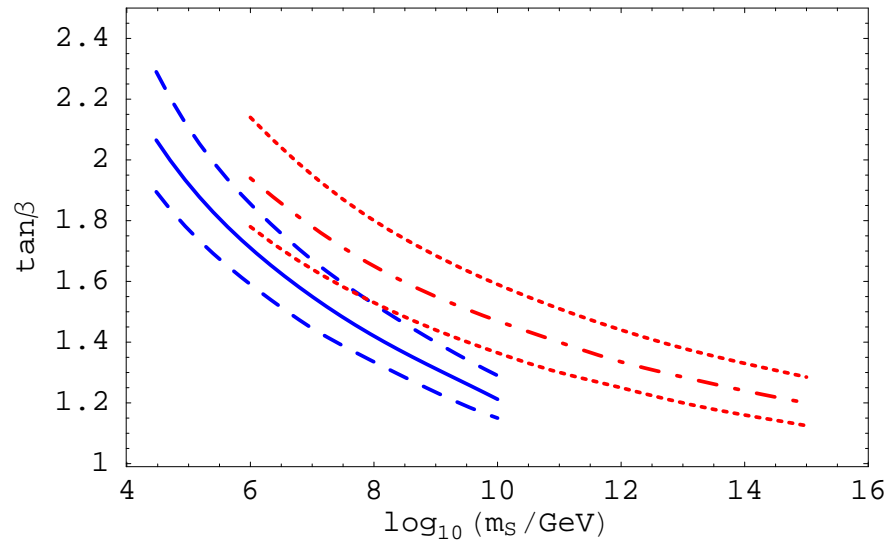

Figure 3: $\tan \beta$ versus the scalar mass scale $m_{S}$ for gauge-top quark Yukawa coupling unification at $M_{\mathrm{GUT}}$. The solid and dashed curves correspond to $M_{3} / M_{1 / 2}=3$, and the dot-dashed and dotted curves to $M_{3} / M_{1 / 2}=10$ for $M_{\text {top }}($ pole $)=172.5 \pm 2.3 \mathrm{GeV}$.

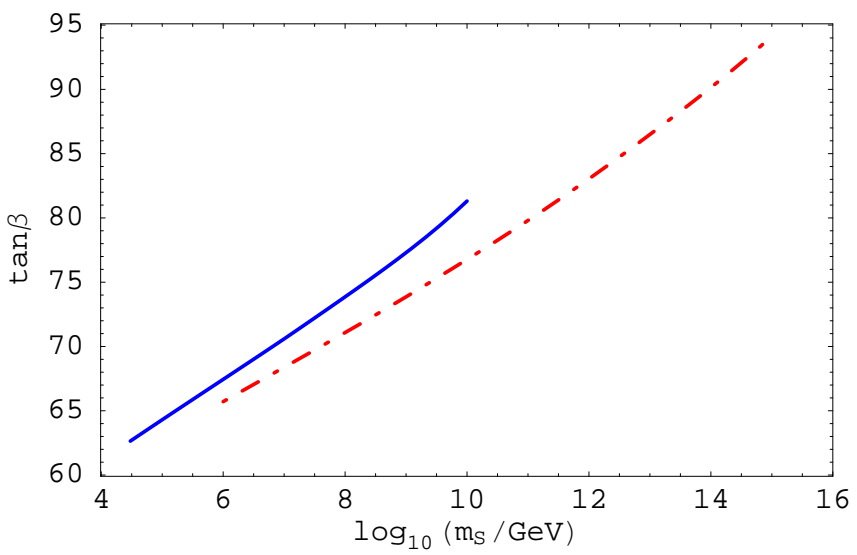

Figure 4: $\tan \beta$ versus the scalar mass scale $m_{S}$ for gauge-bottom quark Yukawa coupling unification at $M_{\mathrm{GUT}}$. The solid curve corresponds to $M_{3} / M_{1 / 2}=3$, and the dotdashed curve to $M_{3} / M_{1 / 2}=10$. The uncertainties in bottom and top quark masses correspond to $5 \%$ and $1 \%$ uncertainties in $\tan \beta$, respectively.

we determine $M_{1 / 2}$ for a given $m_{S}$. Fig. 5 shows $M_{1 / 2}$ for gauge-top quark Yukawa coupling unification, results are similar for gauge-bottom quark or tau lepton Yukawa coupling unification.

The dark matter requirements can be satisfied for $M_{1 / 2}$ ranging from a few hundred $\mathrm{GeV}$ to a few $\mathrm{TeV}$ [8, 14]. It seems possible to satisfy these requirements for any $m_{S}$ from about $1 \mathrm{TeV}$ to $M_{\mathrm{GUT}}$. However, the cosmological effects of a long lived gluino puts an upper bound on $m_{S}$ of $10^{9}$ or $10^{11} \mathrm{GeV}$, where the latter value is for gluino mass less than $300 \mathrm{GeV}$ [15]. Together with gauge coupling unification constraints, this disfavors the case $M_{3} / M_{1 / 2}=10$ in the absence of GUT-scale threshold corrections. $m_{S}$ in the range $10^{6}-10^{11} \mathrm{GeV}$ with $M_{1 / 2} \sim 1 \mathrm{TeV}$ and $M_{3} / M_{1 / 2} \sim 3$ is qualitatively in good agreement with gauge coupling unification and dark matter abundance. 


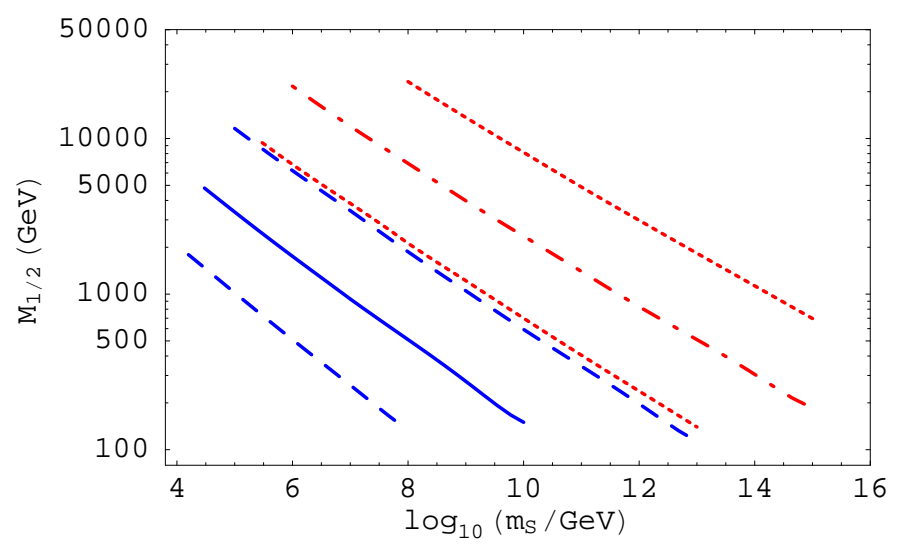

Figure 5: $M_{1 / 2}$ versus $m_{S}$ for gauge-top quark Yukawa coupling unification at $M_{\mathrm{GUT}}$. The solid and dashed curves correspond to $M_{3} / M_{1 / 2}=3$, and the dot-dashed and dotted curves to $M_{3} / M_{1 / 2}=10$ for $\alpha_{s}=0.119 \pm 0.003$.

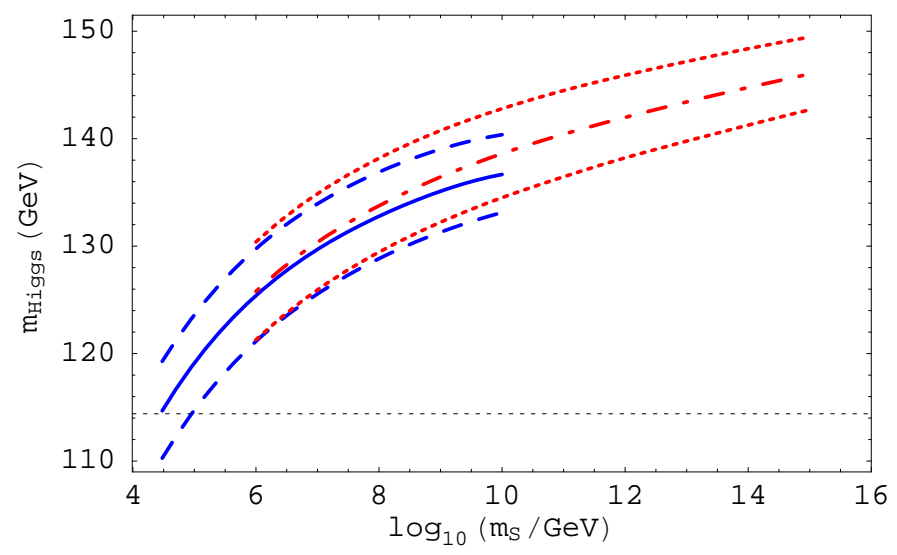

Figure 6: Higgs boson mass $m_{\text {Higgs }}$ versus the scalar mass scale $m_{S}$ for gauge-top quark Yukawa coupling unification at $M_{\mathrm{GUT}}$. The solid and dashed curves correspond to $M_{3} / M_{1 / 2}=3$, and the dot-dashed and dotted curves to $M_{3} / M_{1 / 2}=10$ for $M_{\text {top }}($ pole $)=$ $172.5 \pm 2.3 \mathrm{GeV}$.

The Higgs mass is plotted in Figs. [6 and 7 for gauge-top and gauge-bottom quark Yukawa coupling unification, respectively (Higgs mass is practically identical for gaugebottom quark and gauge-tau lepton Yukawa coupling unification.). Although the main uncertainty in the Higgs boson mass prediction is due to the top quark mass uncertainty, varying gaugino mass can also give a sizable effect. This effect is of order $1 \mathrm{GeV}$ or less for gauge-bottom quark Yukawa coupling unification. However, since the Higgs boson mass is sensitive to $\tan \beta$ for small $\tan \beta$, the effect is larger $(2-3 \mathrm{GeVs})$ for gauge-top quark Yukawa coupling unification. The Higgs boson mass increases as $M_{1 / 2}$ increases, and decreases as the ratio $M_{3} / M_{1 / 2}$ increases (for fixed $M_{1 / 2}$ ).

Because the Higgs boson mass is so sensitive to the top quark Yukawa coupling, it is important to consider the one-loop corrections to the relation between the running $\overline{\mathrm{MS}}$ 


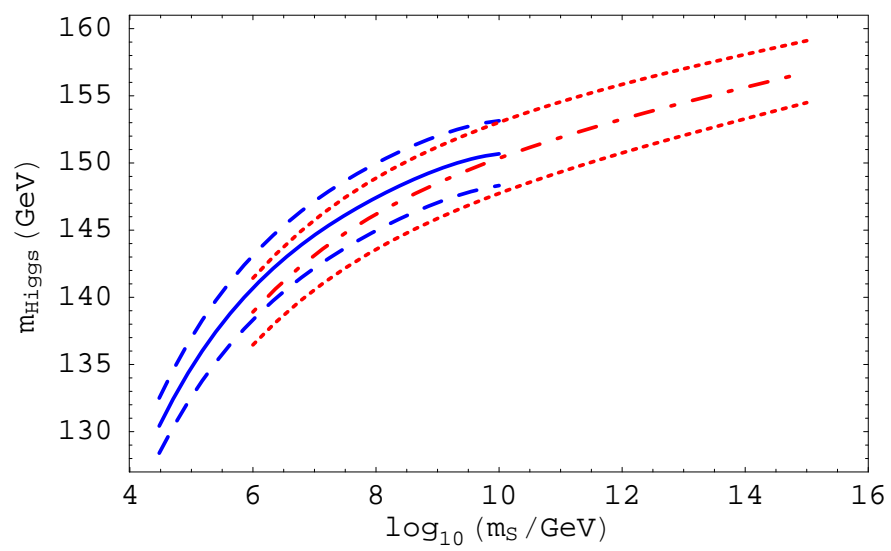

Figure 7: Higgs boson mass $m_{\text {Higgs }}$ versus the scalar mass scale $m_{S}$ for gauge-bottom quark Yukawa coupling unification at $M_{\mathrm{GUT}}$. The solid and dashed curves correspond to $M_{3} / M_{1 / 2}=3$, and the dot-dashed and dotted curves to $M_{3} / M_{1 / 2}=10$ for $M_{\text {top }}($ pole $)=$ $172.5 \pm 2.3 \mathrm{GeV}$.

Yukawa coupling and the pole mass for the top quark. In the numerical calculations we use the results in Ref. [16] (see also Ref. [17] for a review). For the relation between the Higgs boson mass and Higgs quartic coupling, we use the results of Ref. [18, where we choose the renormalization scale to be the top quark pole mass [19. We do not consider the split SUSY corrections to these threshold effects. These were considered in Ref. [20] and were found to be less important than the SM corrections, generally affecting the Higgs mass $\lesssim 2 \mathrm{GeV}$.

\section{Conclusions}

We have constructed a class of 7D orbifold GUTs with $\mathcal{N}=1$ supersymmetry in which the SM gauge couplings and the top quark (bottom quark or tau lepton) Yukawa coupling are unified at the GUT scale. Assuming split supersymmetry, we can reliably estimate the SM Higgs boson mass. For gauge-top quark Yukawa coupling unification with $10^{6} \mathrm{GeV} \lesssim m_{S} \lesssim 10^{11} \mathrm{GeV}$, the Higgs boson mass range is $131 \pm 10 \mathrm{GeV}$ for $M_{\text {top }}($ pole $)=172.5 \pm 2.3 \mathrm{GeV}$. For gauge-bottom quark (or tau lepton) Yukawa coupling unification, the Higgs boson mass range is $146 \pm 8 \mathrm{GeV}$.

\section{Acknowledgments}

This work is supported in part by DOE Grant \# DE-FG02-84ER40163 (I.G.), \#DEFG02-96ER40959 (T.L.), \# DE-FG02-91ER40626 (Q.S. and V.N.S.), and by a University of Delaware graduate fellowship (V.N.S.). 


\section{Appendix A: Seven-Dimensional Orbifold Models}

We consider a 7D space-time $M^{4} \times T^{2} / Z_{6} \times S^{1} / Z_{2}$ with coordinates $x^{\mu},(\mu=0,1,2,3)$, $x^{5}, x^{6}$ and $x^{7}$. The torus $T^{2}$ is homeomorphic to $S^{1} \times S^{1}$ and the radii of the circles along the $x^{5}, x^{6}$ and $x^{7}$ directions are $R_{1}, R_{2}$, and $R^{\prime}$, respectively. We define the complex coordinate $z$ for $T^{2}$ and the real coordinate $y$ for $S^{1}$,

$$
z \equiv \frac{1}{2}\left(x^{5}+i x^{6}\right), \quad y \equiv x^{7} .
$$

The torus $T^{2}$ can be defined by $C^{1}$ modulo the equivalent classes:

$$
z \sim z+\pi R_{1}, \quad z \sim z+\pi R_{2} e^{\mathrm{i} \theta}
$$

To obtain the orbifold $T^{2} / Z_{6}$, we require that $R_{1}=R_{2} \equiv R$ and $\theta=\pi / 3$. Then $T^{2} / Z_{6}$ is obtained from $T^{2}$ by moduloing the equivalent class

$$
\Gamma_{T}: \quad z \sim \omega z
$$

where $\omega=e^{\mathrm{i} \pi / 3}$. There is one $Z_{6}$ fixed point $z=0$, two $Z_{3}$ fixed points: $z=\pi R e^{\mathrm{i} \pi / 6} / \sqrt{3}$ and $z=2 \pi R e^{\mathrm{i} \pi / 6} / \sqrt{3}$, and three $Z_{2}$ fixed points: $z=\sqrt{3} \pi R e^{\mathrm{i} \pi / 6} / 2, z=\pi R / 2$ and $z=\pi R e^{\mathrm{i} \pi / 3} / 2$. The orbifold $S^{1} / Z_{2}$ is obtained from $S^{1}$ by moduloing the equivalent class

$$
\Gamma_{S}: \quad y \sim-y
$$

There are two fixed points: $y=0$ and $y=\pi R^{\prime}$. The $\mathcal{N}=1$ supersymmetry in 7D has 16 supercharges corresponding to $\mathcal{N}=4$ supersymmetry in $4 \mathrm{D}$, and only the gauge multiplet can be introduced in the bulk. This multiplet can be decomposed under 4D $\mathcal{N}=1$ supersymmetry into a gauge vector multiplet $V$ and three chiral multiplets $\Sigma_{1}$, $\Sigma_{2}$, and $\Sigma_{3}$ in the adjoint representation, where the fifth and sixth components of the gauge field, $A_{5}$ and $A_{6}$, are contained in the lowest component of $\Sigma_{1}$, and the seventh component of the gauge field $A_{7}$ is contained in the lowest component of $\Sigma_{2}$.

We express the bulk action in the Wess-Zumino gauge and $4 \mathrm{D} \mathcal{N}=1$ supersymmetry notation [5]

$$
\begin{aligned}
\mathcal{S}= & \int d^{7} x\left\{\operatorname { T r } \left[\int d^{2} \theta\left(\frac{1}{4 k g^{2}} \mathcal{W}^{\alpha} \mathcal{W}_{\alpha}+\frac{1}{k g^{2}}\left(\Sigma_{3} \partial_{z} \Sigma_{2}+\Sigma_{1} \partial_{y} \Sigma_{3}-\frac{1}{\sqrt{2}} \Sigma_{1}\left[\Sigma_{2}, \Sigma_{3}\right]\right)\right)\right.\right. \\
& + \text { h.c. }]+\int d^{4} \theta \frac{1}{k g^{2}} \operatorname{Tr}\left[\left(\sqrt{2} \partial_{z}^{\dagger}+\Sigma_{1}^{\dagger}\right) e^{-V}\left(-\sqrt{2} \partial_{z}+\Sigma_{1}\right) e^{V}+\partial_{z}^{\dagger} e^{-V} \partial_{z} e^{V}\right. \\
& \left.\left.+\left(\sqrt{2} \partial_{y}+\Sigma_{2}^{\dagger}\right) e^{-V}\left(-\sqrt{2} \partial_{y}+\Sigma_{2}\right) e^{V}+\partial_{y} e^{-V} \partial_{y} e^{V}+\Sigma_{3}^{\dagger} e^{-V} \Sigma_{3} e^{V}\right]\right\}
\end{aligned}
$$


where $k$ is the normalization of the group generator, and $\mathcal{W}_{\alpha}$ denotes the gauge field strength. From the above action, we obtain the transformations of the vector multiplet:

$$
\begin{aligned}
V\left(x^{\mu}, \omega z, \omega^{-1} \bar{z}, y\right) & =R_{\Gamma_{T}} V\left(x^{\mu}, z, \bar{z}, y\right) R_{\Gamma_{T}}^{-1}, \\
\Sigma_{1}\left(x^{\mu}, \omega z, \omega^{-1} \bar{z}, y\right) & =\omega^{-1} R_{\Gamma_{T}} \Sigma_{1}\left(x^{\mu}, z, \bar{z}, y\right) R_{\Gamma_{T}}^{-1} \\
\Sigma_{2}\left(x^{\mu}, \omega z, \omega^{-1} \bar{z}, y\right) & =R_{\Gamma_{T}} \Sigma_{2}\left(x^{\mu}, z, \bar{z}, y\right) R_{\Gamma_{T}}^{-1}, \\
\Sigma_{3}\left(x^{\mu}, \omega z, \omega^{-1} \bar{z}, y\right) & =\omega R_{\Gamma_{T}} \Sigma_{3}\left(x^{\mu}, z, \bar{z}, y\right) R_{\Gamma_{T}}^{-1} \\
V\left(x^{\mu}, z, \bar{z},-y\right) & =R_{\Gamma_{S}} V\left(x^{\mu}, z, \bar{z}, y\right) R_{\Gamma_{S}}^{-1}, \\
\Sigma_{1}\left(x^{\mu}, z, \bar{z},-y\right) & =R_{\Gamma_{S} \Sigma_{1}}\left(x^{\mu}, z, \bar{z}, y\right) R_{\Gamma_{S}}^{-1} \\
\Sigma_{2}\left(x^{\mu}, z, \bar{z},-y\right) & =-R_{\Gamma_{S}} \Sigma_{2}\left(x^{\mu}, z, \bar{z}, y\right) R_{\Gamma_{S}}^{-1} \\
\Sigma_{3}\left(x^{\mu}, z, \bar{z},-y\right) & =-R_{\Gamma_{S}} \Sigma_{3}\left(x^{\mu}, z, \bar{z}, y\right) R_{\Gamma_{S}}^{-1}
\end{aligned}
$$

where we introduce non-trivial transformation $R_{\Gamma_{T}}$ and $R_{\Gamma_{S}}$ to break the bulk gauge group $G$.

The $Z_{6} \times Z_{2}$ transformation properties for the decomposed components of $V, \Sigma_{1}$, $\Sigma_{2}$, and $\Sigma_{3}$ in our $S U(7)$ models are given by

$$
\begin{gathered}
V:\left(\begin{array}{cccc}
(1,+) & \left(\omega^{-n_{1}},+\right) & \left(\omega^{-n_{1}},-\right) & \left(\omega^{-n_{2}},-\right) \\
\left(\omega^{n_{1}},+\right) & (1,+) & (1,-) & \left(\omega^{n_{1}-n_{2}},-\right) \\
\left(\omega^{n_{1}},-\right) & (1,-) & (1,+) & \left(\omega^{n_{1}-n_{2}},+\right) \\
\left(\omega^{n_{2}},-\right) & \left(\omega^{n_{2}-n_{1}},-\right) & \left(\omega^{n_{2}-n_{1}},+\right) & (1,+)
\end{array}\right)+(1,+), \\
\Sigma_{1}:\left(\begin{array}{cccc}
\left(\omega^{-1},+\right) & \left(\omega^{-n_{1}-1},+\right) & \left(\omega^{-n_{1}-1},-\right) & \left(\omega^{-n_{2}-1},-\right) \\
\left(\omega^{n_{1}-1},+\right) & \left(\omega^{-1},+\right) & \left(\omega^{-1},-\right) & \left(\omega^{n_{1}-n_{2}-1},-\right) \\
\left(\omega^{n_{1}-1},-\right) & \left(\omega^{-1},-\right) & \left(\omega^{-1},+\right) & \left(\omega^{n_{1}-n_{2}-1},+\right) \\
\left(\omega^{n_{2}-1},-\right) & \left(\omega^{n_{2}-n_{1}-1},-\right) & \left(\omega^{n_{2}-n_{1}-1},+\right) & \left(\omega^{-1},+\right)
\end{array}\right)+\left(\omega^{-1},+\right),
\end{gathered}
$$




$$
\begin{gathered}
\Sigma_{2}:\left(\begin{array}{cccc}
(1,-) & \left(\omega^{-n_{1}},-\right) & \left(\omega^{-n_{1}},+\right) & \left(\omega^{-n_{2}},+\right) \\
\left(\omega^{n_{1}},-\right) & (1,-) & (1,+) & \left(\omega^{n_{1}-n_{2}},+\right) \\
\left(\omega^{n_{1}},+\right) & (1,+) & (1,-) & \left(\omega^{n_{1}-n_{2}},-\right) \\
\left(\omega^{n_{2}},+\right) & \left(\omega^{n_{2}-n_{1}},+\right) & \left(\omega^{n_{2}-n_{1}},-\right) & (1,-)
\end{array}\right)+(1,-), \\
\Sigma_{3}:\left(\begin{array}{cccc}
(\omega,-) & \left(\omega^{-n_{1}+1},-\right) & \left(\omega^{-n_{1}+1},+\right) & \left(\omega^{-n_{2}+1},+\right) \\
\left(\omega^{n_{1}+1},-\right) & (\omega,-) & (\omega,+) & \left(\omega^{n_{1}-n_{2}+1},+\right) \\
\left(\omega^{n_{1}+1},+\right) & (\omega,+) & (\omega,-) & \left(\omega^{n_{1}-n_{2}+1},-\right) \\
\left(\omega^{n_{2}+1},+\right) & \left(\omega^{n_{2}-n_{1}+1},+\right) & \left(\omega^{n_{2}-n_{1}+1},-\right) & (\omega,-)
\end{array}\right)+(\omega,-),
\end{gathered}
$$

where the zero modes transform as $(1,+)$.

From Eqs. (36)-(39), we find that the $7 \mathrm{D} \mathcal{N}=1$ supersymmetric gauge symmetry $S U(7)$ is broken down to $4 \mathrm{D} \mathcal{N}=1$ supersymmetric gauge symmetry $S U(3)_{C} \times$ $S U(2)_{L} \times U(1)_{Y} \times U(1)_{\alpha} \times U(1)_{\beta}$ [4]. In addition, there are zero modes from the chiral multiplets $\Sigma_{1}, \Sigma_{2}$ and $\Sigma_{3}$ which play an important role in gauge-Yukawa coupling unification 3 .

\section{References}

[1] I. Gogoladze, T. Li and Q. Shafi, Phys. Rev. D 73, 066008 (2006).

[2] V. Barger, J. Jiang, P. Langacker and T. Li, Phys. Lett. B 624, 233 (2005); Nucl. Phys. B 726, 149 (2005).

[3] I. Gogoladze, Y. Mimura and S. Nandi, Phys. Lett. B 562, 307 (2003); Phys. Rev. Lett. 91, 141801 (2003); Phys. Rev. D 69, 075006 (2004); I. Gogoladze, Y. Mimura, S. Nandi and K. Tobe, Phys. Lett. B 575, 66 (2003); I. Gogoladze, T. Li, Y. Mimura and S. Nandi, Phys. Lett. B 622, 320 (2005); Phys. Rev. D 72, 055006 (2005).

[4] T. Li, Phys. Lett. B 520, 377 (2001); Nucl. Phys. B 619, 75 (2001); Nucl. Phys. B 633, 83 (2002).

[5] N. Marcus, A. Sagnotti and W. Siegel, Nucl. Phys. B 224, 159 (1983); N. ArkaniHamed, T. Gregoire and J. Wacker, JHEP 0203, 055 (2002).

[6] Y. Kawamura, Prog. Theor. Phys. 103, 613 (2000); 105, 999 (2001); G. Altarelli and F. Feruglio, Phys. Lett. B 511, 257 (2001); A. B. Kobakhidze, Phys. Lett. B 514, 131 (2001); L. Hall and Y. Nomura, Phys. Rev. D 64, 055003 (2001); A. Hebecker and J. March-Russell, Nucl. Phys. B 613, 3 (2001). 
[7] N. Arkani-Hamed and S. Dimopoulos, JHEP 0506, 073 (2005).

[8] G. F. Giudice and A. Romanino, Nucl. Phys. B 699, 65 (2004) [Erratum-ibid. B 706, 65 (2005)].

[9] A. Arvanitaki, C. Davis, P. W. Graham, and J. G. Wacker, Phys. Rev. D 70, 117703 (2004).

[10] S. Eidelman et al. [Particle Data Group Collab.], Phys. Lett. B 592, (2004) 1.

[11] [Tevatron Electroweak Working Group], hep-ex/0603039.

[12] P. C. Schuster, JHEP 0601, 148 (2006).

[13] B. Dutta and Y. Mimura, Phys. Lett. B 627, 145 (2005).

[14] A. Pierce, Phys. Rev. D 70, 075006 (2004).

[15] A. Arvanitaki, C. Davis, P. W. Graham, A. Pierce, and J. G. Wacker, Phys. Rev. D 72, 075011 (2005).

[16] N. Gray, D. J. Broadhurst, W. Grafe and K. Schilcher, Z. Phys. C 48, 673 (1990);

R. Hempfling and B. A. Kniehl, Phys. Rev. D 51, 1386 (1995).

[17] B. Schrempp and M. Wimmer, Prog. Part. Nucl. Phys. 37, 1 (1996).

[18] A. Sirlin and R. Zucchini, Nucl. Phys. B 266, 389 (1986).

[19] T. Hambye and K. Riesselmann, Phys. Rev. D 55, 7255 (1997).

[20] M. Binger (2004), hep-ph/0408240. 